\title{
Ventilatory Efficiency Before and After Lung Volume Reduction Surgery
}

\author{
Hilary F Armstrong MA, Nicole E Dussault, Wilawan Thirapatarapong MD, \\ Renee S Lemieux, Byron M Thomashow MD, and Matthew N Bartels MD
}

\begin{abstract}
BACKGROUND: Lung volume reduction surgery (LVRS) has been shown to improve the clearance of carbon dioxide and minute ventilation $\left(\dot{\mathrm{V}}_{\mathbf{E}}\right)$ in select patients with COPD. One variable often assessed in COPD is ventilatory efficiency $\left(\dot{\mathrm{V}}_{\mathrm{E}} / \dot{\mathrm{V}}_{\mathrm{CO}}\right)$. METHODS: We compared 55 LVRS subjects with 25 controls from the National Emphysema Treatment Trial. $\dot{\mathrm{V}}_{\mathrm{E}} / \dot{\mathrm{V}}_{\mathrm{CO}_{2}}$ was calculated from cardiopulmonary exercise testing at baseline and 6-months. We sought to assess $\dot{\mathrm{V}}_{\mathbf{E}} / \dot{\mathrm{V}}_{\mathrm{CO}_{2}}$ changes with LVRS compared with controls who only received standard medical care. RESULTS: At 6 months, the LVRS group significantly increased peak $\dot{\mathrm{V}}_{\mathrm{O}_{2}}$, work load, $\dot{\mathrm{V}}_{\mathrm{E}}, \dot{\mathrm{V}}_{\mathrm{CO}_{2}}$, and tidal volume while lowering peak and lowest $\dot{\mathrm{V}}_{\mathrm{E}} / \dot{\mathrm{V}}_{\mathbf{C O}_{2}}$ (improved ventilatory efficiency) and end-tidal carbon dioxide pressure. The control group did not display these changes. The changes were greatest in the LVRS subjects who improved their exercise capacity after surgery ( $>10 \mathrm{~W}$ ). CONCLUSIONS: The changes were greatest in the LVRS subjects who showed the most functional improvement, indicating an association of improved ventilation with improved functional outcome. Key words: chronic obstructive pulmonary disease; exercise; gas exchange. [Respir Care 2015;60(1):63-71. (C) 2015 Daedalus Enterprises]
\end{abstract}

\section{Introduction}

One variable that is often used to assess the exercise capacity in patients with COPD is ventilatory efficiency, calculated by the relationship between minute ventilation $\left(\dot{\mathrm{V}}_{\mathrm{E}}\right)$ and the rate of carbon dioxide production $\left(\dot{\mathrm{V}}_{\mathrm{CO}_{2}}\right)$ : $\dot{\mathrm{V}}_{\mathrm{E}} / \dot{\mathrm{V}}_{\mathrm{CO}_{2}}$. Peak $\dot{\mathrm{V}}_{\mathrm{E}} / \dot{\mathrm{V}}_{\mathrm{CO}_{2}}$ has been found to predict mortal-

\footnotetext{
Ms Armstrong, Ms Dussault, Dr Thirapatarapong, Ms Lemieux, and Dr Bartels are affiliated with the Department of Rehabilitation and Regenerative Medicine, Columbia University Medical Center, New York, New York; Ms Armstrong is affiliated with the Department of Epidemiology, Mailman School of Public Health, Columbia University, New York, New York; Dr Thirapatarapong is affiliated with the Department of Rehabilitation Medicine, Faculty of Medicine Siriraj Hospital, Mahidol University, Bangkok, Thailand; Dr Thomashow is affiliated with the Department of Medicine: Pulmonary, Allergy and Critical Care, Columbia University Medical Center, New York, New York; Dr Bartels is affiliated with the Department of Rehabilitation Medicine, Albert Einstein College of Medicine/Montefiore Medical Center, Bronx, New York.
}

The authors have disclosed no conflicts of interest.

This research was funded in part by the Vidda Foundation. This publication was supported by grant UL1 TR000040 from the National Center for Advancing Translational Sciences, National Institutes of Health. ity better than oxygen consumption $\left(\dot{\mathrm{V}}_{\mathrm{O}_{2}}\right)$ in elderly patients with heart failure. ${ }^{1}$ Additionally, $\dot{\mathrm{V}}_{\mathrm{E}} / \dot{\mathrm{V}}_{\mathrm{CO}_{2}}$ slope has been shown to outperform $\dot{\mathrm{V}}_{\mathrm{O}_{2}}$ as a predictor of respiratory complications and mortality in patients after pulmonary resection, ${ }^{2}$ increased mortality in patients with COPD undergoing surgery for lung cancer, ${ }^{3}$ and mortality prognostication in heart failure. ${ }^{4-8}$ In COPD patients, the $\dot{\mathrm{V}}_{\mathrm{E}} / \dot{\mathrm{V}}_{\mathrm{CO}_{2}}$ slope was found to be negatively related to peak $\dot{\mathrm{V}}_{\mathrm{O}_{2}}{ }^{9,10}$ Furthermore, $\dot{\mathrm{V}}_{\mathrm{E}} / \dot{\mathrm{V}}_{\mathrm{CO}_{2}}$ was shown to be inversely proportional to COPD severity, indicating altered gas exchange between patients with various severities of COPD. ${ }^{11}$ Even the intercept of $\dot{\mathrm{V}}_{\mathrm{E}} / \dot{\mathrm{V}}_{\mathrm{CO}_{2}}$ has been found to be associated with the ventilatory response during exercise ${ }^{10,12}$ However, there has been no thorough investigation regarding the change in ventilatory efficiency before and after most surgical interventions in patients with COPD since the National Emphysema Treatment Trial (NETT). ${ }^{13}$

Correspondence: Hilary F Armstrong, MA, 180 Fort Washington Avenue, HP 1-169b, New York, NY 10032. E-mail: hfa2104@columbia.edu.

DOI: $10.4187 /$ respcare. 03233 
COPD is now the third leading cause of death in the United States ${ }^{14}$ and by 2030 is expected to rank third in worldwide burden of disease, according to a study published by the World Bank/World Health Organization. ${ }^{15}$ Lung volume reduction surgery (LVRS) has been shown to be an effective surgical option for a subset of patients with severe emphysema. In appropriately selected patients, LVRS can decrease morbidity, mortality, ${ }^{13,16}$ and dyspnea, ${ }^{17-19}$ while improving exercise performance, ${ }^{20,21}$ endurance, ${ }^{22}$ pulmonary function, ${ }^{20}$ and quality of life.$^{21}$ The NETT used improvement in exercise capacity as one of the main outcomes in their federally sponsored, multicenter, randomized clinical trial that evaluated the effectiveness and safety of LVRS versus standard treatment. ${ }^{13}$

Cardiopulmonary exercise testing (CPET) measures a broad range of variables related to cardiorespiratory function and links metabolic, cardiovascular, and pulmonary responses to exercise. ${ }^{23}$ One of these variables is ventilatory efficiency, which represents the effectiveness of pulmonary clearance of carbon dioxide during exercise. ${ }^{24,25}$ During CPET, ventilatory efficiency is usually calculated as the ratio of $\dot{\mathrm{V}}_{\mathrm{E}}$ to $\dot{\mathrm{V}}_{\mathrm{CO}_{2}}$. In COPD, abnormal lung mechanics often limit the capacity for $\dot{\mathrm{V}}_{\mathrm{E}}$. Because it is known that LVRS improves the clearance of $\mathrm{CO}_{2}{ }^{26-28}$ and $\dot{V}_{\mathrm{E}}$ in patients with COPD, ${ }^{26,27}$ we hypothesized that there would be an improvement in ventilatory efficiency after LVRS. A previous study has assessed the impact of ventilatory efficiency's association with weight gain ${ }^{29}$ and showed that weight gain after LVRS was related to improved lung function and ventilatory efficiency. Exercise capacity and gas exchange (not including $\dot{\mathrm{V}}_{\mathrm{E}} / \dot{\mathrm{V}}_{\mathrm{CO}_{2}}$ ) after LVRS have been studied. ${ }^{13,26,27,30}$ Additionally, NETT trial papers have only reported arterial levels of $\mathrm{CO}_{2}$ instead of $\mathrm{V}_{\mathrm{CO}_{2}}{ }^{26}$ so ventilatory efficiency could not be assessed directly. Because of this lack of thorough investigation regarding the change in ventilatory efficiency before and after LVRS in COPD, we sought to assess $\dot{\mathrm{V}}_{\mathrm{E}} / \dot{\mathrm{V}}_{\mathrm{CO}_{2}}$ in our LVRS subjects.

\section{Methods}

\section{Subjects}

The institutional review board of the New York Presbyterian-Columbia University Medical Center (New York, New York) approved this study. A retrospective chart review was performed on all patients who underwent LVRS at Columbia University Medical Center from January 1998 to October 2009. Subjects who had CPET and pulmonary function tests (PFT) within 2 months $(37 \pm 27 \mathrm{~d})$ before LVRS and repeat testing approximately 6 months (191 \pm 26 d) after were included. PFTs concurrent with CPET were chosen. The subjects underwent 16-20 sessions (minimum) of pulmonary rehabilitation over 10-12 weeks, performed the first CPET 2 months before LVRS

\section{QUICK LOOK}

\section{Current knowledge}

COPD represents a significant worldwide public health burden and is a leading cause of morbidity and mortality. Lung volume reduction surgery has been demonstrated to reduce hypercarbia and minute ventilation in select subjects with the disease.

\section{What this paper contributes to our knowledge}

Following lung volume reduction surgery, ventilatory efficiency improves as measured by the peak minute ventilation/carbon dioxide production and lowest minute ventilation/carbon dioxide production during cardiopulmonary exercise testing. The greatest improvements were seen in subjects who increased their peak wattage by greater than $10 \mathrm{~W}$ from baseline to 6 months after surgery. These changes suggest an increase in alveolar ventilation after lung volume reduction.

(baseline), and underwent a repeated 10-12 weeks of 16-20 sessions after surgery rehabilitation. These subjects then performed a second CPET 6 months after LVRS. These rehabilitation protocols are still used at this center for surgical patients, even after discontinuation of the NETT. The selection criteria after the completion of the NETT are according to National Coverage Determination Centers for Medicare \& Medicaid Services guidelines for LVRS. ${ }^{31}$ In brief, subjects in this study of LVRS have Global Initiative for Chronic Obstructive Lung Disease level 3 or 4 COPD with severe exercise limitation (peak wattage less than $40 \mathrm{~W}$ for men and $25 \mathrm{~W}$ for women, with preference for upper lobe disease in Centers for Medicare \& Medicaid Services guidelines, severe emphysema in NETT). Additionally, subjects had no pulmonary hypertension and no evidence of active cardiac disease.

To rule out the possible effects of the intensive pre- and post-LVRS pulmonary rehabilitation given to the subjects, and to have a control group of comparable subjects eligible for LVRS, we analyzed the NETT subjects who were randomized to the non-surgical group (they underwent only pulmonary rehabilitation both before and after randomization) at our center between 1998 and 2003. Full exercise programs, as prescribed in the NETT, consisted of 16-20 sessions over 10 weeks, with a similar program after randomization. $^{32}$

Medications, specifically cardioactive medications such as beta agonists, beta blockers, anticholinergics, digitalis, and calcium channel blockers, were reviewed in each subject's chart for both the first and second CPET. Additionally, a dobutamine stress echocardiogram or thallium testing was performed to assess for ischemic heart disease. 
Diagnosis for subjects with any suspicious changes on the dobutamine or thallium test was confirmed with cardiac catheterization. Left ventricular ejection fraction $(>45 \%)$ and absence of significant valvular disease were confirmed by review of the echocardiogram closest to the time of the first CPET.

\section{Pulmonary Function and Exercise Testing}

All PFTs were performed according to the NETT protocol. ${ }^{13}$ All values are reported post-bronchodilator, as per NETT protocol. This includes FVC, $\mathrm{FEV}_{1}$, total lung capacity (TLC), and residual volume (RV). Percent of predicted PFTs were calculated for males and females as described in prior publications. ${ }^{33-35}$

CPET was performed on an electronically braked cycle ergometer (Ergometrics 800, SensorMedics, Yorba Linda, California) with a Viasys SensorMedics Encore metabolic cart (Viasys Corporation, Loma Linda, California); before 2005, a Vmax 229 series workstation (SensorMedics) was used. The equipment was calibrated before every test. Continuous 12-lead telemetry was monitored via CardioSoft electrocardiogram software (GE/CardioSoft, Houston, Texas); before 2005, a model Max-1 electrocardiogram was used (Marquette Medical Systems, Milwaukee, Wisconsin). Oxygen saturation was recorded with a N595 pulse oximeter (Nellcor, Boulder, Colorado); before 2005, a SensorMedics Sat-Trak (SensorMedics) was used. All subjects were tested on $29.99 \pm 0.25 \%$ fractional inspired oxygen via a closed system both before and after LVRS; all calibrations were 2-point calibrations with hyperoxic and hypoxic points and included hypercarbic and absent carbon dioxide levels of calibration. The metabolic system was validated for use with hyperoxic testing and was stable for measurement of $\mathrm{FeCO}_{2}$ at hyperoxic situations. The exercise protocol was ramping and followed the NETT $^{13}$ and American Thoracic Society guidelines. ${ }^{36,37}$ Even after termination of the NETT, NETT guidelines were followed at this institution for CPET. An individualized ramping protocol was determined by the maximal voluntary ventilation (MVV) test. The MVV was performed over $12 \mathrm{~s}$. Individuals who achieved $\leq 40 \mathrm{~L} / \mathrm{min} \mathrm{MVV}$ performed a $5-\mathrm{W} / \mathrm{min}$ ramping protocol, whereas those attaining $>40 \mathrm{~L} / \mathrm{min}$ MVV performed a $10-\mathrm{W}$ ramping protocol. The same ramp protocol was used pre- and postLVRS or at both sessions in the non-surgical group. CPET variables were collected breath-by-breath, and included rate of carbon dioxide production $\left(\dot{\mathrm{V}}_{\mathrm{CO}_{2}}\right)$, maximal work load (peak watts), volume of oxygen consumption by weight $\left(\dot{\mathrm{V}}_{\mathrm{O}_{2}}\right.$ in $\left.\mathrm{mL} / \mathrm{kg} / \mathrm{min}\right)$, percent-of-predicted oxygen consumption attained $\left(\dot{\mathrm{V}}_{\mathrm{O}_{2}}[\%\right.$ pred] $), \dot{\mathrm{V}}_{\mathrm{E}}$, tidal volume $\left(\mathrm{V}_{\mathrm{T}}\right)$, breathing frequency, partial pressure of end-tidal $\mathrm{CO}_{2}$ $\left(\mathrm{P}_{\mathrm{ETCO}_{2}}\right), \mathrm{HR}$ at baseline $\left(\mathrm{HR}_{\text {rest }}\right)$, and heart rate at peak exercise $\left(\mathrm{HR}_{\max }\right)$. Peak variables averaged over $20 \mathrm{~s}$ in- clude $\dot{\mathrm{V}}_{\mathrm{O}_{2}}, \dot{\mathrm{V}}_{\mathrm{E}}, \dot{\mathrm{V}}_{\mathrm{CO}_{2}}, \mathrm{P}_{\mathrm{ETCO}_{2}}$, and $\mathrm{V}_{\mathrm{T}}$. Ventilatory efficiency was measured in 4 ways: (1) the $\dot{\mathrm{V}}_{\mathrm{E}} / \dot{\mathrm{V}}_{\mathrm{CO}_{2}}$ slope, taken as the linear relationship between $\dot{\mathrm{V}}_{\mathrm{E}}$ and $\dot{\mathrm{V}}_{\mathrm{CO}_{2}} ;$; (2) the intercept of the $\dot{\mathrm{V}}_{\mathrm{E}} / \dot{\mathrm{V}}_{\mathrm{CO}_{2}}$ regression line; (3) the lowest $\dot{\mathrm{V}}_{\mathrm{E}} / \dot{\mathrm{V}}_{\mathrm{CO}_{2}}$ ratio during exercise; and (4) the $\dot{\mathrm{V}}_{\mathrm{E}} / \dot{\mathrm{V}}_{\mathrm{CO}_{2}}$ at peak exercise. The $\dot{\mathrm{V}}_{\mathrm{E}} / \dot{\mathrm{V}}_{\mathrm{CO}_{2}}$ slope was calculated using the breath-by-breath data averaged over $20 \mathrm{~s}$. Rest was not included in the slope calculation; the 3-min warm-up was included.

Age-predicted peak HR was calculated using $208-(0.7$ $\times$ age). ${ }^{38}$ The percent-of-predicted heart rate reserve (\%HRR) was calculated as $\% \mathrm{HRR}=\left(\mathrm{HR}_{\max }-\mathrm{HR}_{\text {rest }}\right) /([$ age predicted HR]) $\left.-\mathrm{HR}_{\text {rest }}\right) \times 100 .{ }^{39}$ Breathing reserve was calculated as $100 \times\left(\mathrm{MVV}-\right.$ peak $\left.\dot{\mathrm{V}}_{\mathrm{E}}\right) / \mathrm{MVV}$. $\dot{\mathrm{V}}_{\mathrm{O}_{2}}(\%$ pred $)$ was calculated from the 1983 Jones equation for males as $4.2-(0.032 \times$ age $)$ and for females as $2.6-(0.014 \times$ age $)$.

\section{Statistics}

Data were analyzed with SPSS 20 (SPSS, Chicago, Illinois). Variables are presented as mean \pm SD. Continuous variables were analyzed with a paired-sample $t$ test to evaluate whether there were differences between baseline and 6 months. Only subjects with complete data were used. An independent-sample $t$ test was used to assess differences between those included and not included and between the non-surgical group and the surgical group. Simple linear regression was used to determine the association between variables. Chi-square test was used to determine differences between categorical variables. Statistical significance was set a priori at $P \leq .05$.

It was decided, in addition, to compare the LVRS subjects who significantly improved their exercise capacity after surgery with those who did not. An improvement in maximal exercise capacity was defined as an increase in the maximal work load of $>10 \mathrm{~W}$ from the baseline level, as defined from the results in the NETT. ${ }^{13}$ We explored whether the changes in functional improvement would be associated with the changes in ventilatory improvement. Furthermore, an iso-work load analysis was used to compare the same work loads at both exercise tests. The maximal work load achieved by the subject during either the baseline or 6-month time period was chosen for comparison. For example, if the maximal work load at baseline was $15 \mathrm{~W}$, then $15 \mathrm{~W}$ was the level used for both tests to compare CPET variables. Looking at iso-work load allowed us to control for the increase in functional capacity.

\section{Results}

\section{Baseline Demographics and Pulmonary Function}

Figure 1 shows a flow chart of the data. Of the 125 subjects who underwent LVRS between 1998 and 2009, 


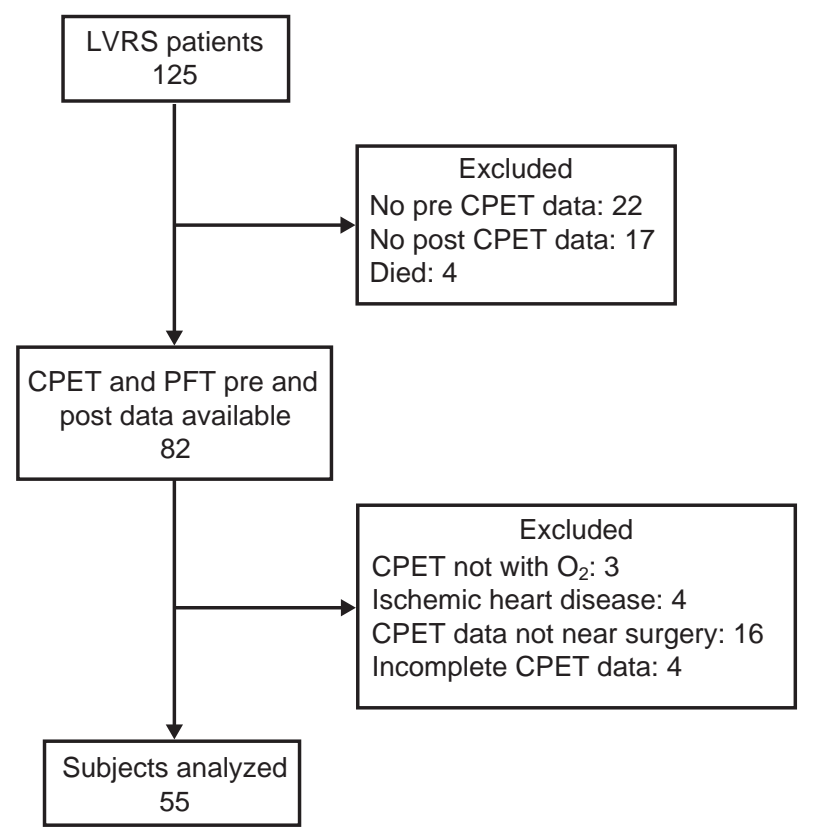

Fig. 1. Flow chart. LVRS = lung volume reduction surgery, $\mathrm{CPET}=$ cardiopulmonary exercise test, PFT = pulmonary function test.

82 subjects had PFT and CPET data before and after LVRS. Despite our usual practice of performing CPET both before and after surgery to assess function and help guide exercise programs, there was variability in practice before the NETT guidelines were established in 2004. Twentytwo subjects had no CPET before LVRS, 17 subjects had no CPET after LVRS due to loss of follow up, and 4 subjects died within 6 months of surgery. Three additional subjects were excluded because the CPET before LVRS was done on room air instead of supplemental oxygen, 4 had ischemic heart disease, and an additional 16 were excluded because the CPET was too far from the time of surgery. Four subjects did not have completed data. Overall, 55 subjects were available for analysis. Additionally, 25 controls (non-surgical group) were analyzed.

All subjects were former heavy smokers who were confirmed abstinent from smoking for at least 6 months before and after surgery. All subjects had an electrocardiogram at the time of CPET and were in sinus rhythm. None had active ischemic heart disease. In comparison with the surgery group, there were a greater proportion of men in the non-surgical group. All other demographics and pulmonary function variables were comparable between the two groups at baseline (Table 1). Medications did not significantly differ between LVRS subjects or the non-surgical group before and after surgery or rehabilitation. Specifically, regarding cardioactive medications, all subjects were on a beta agonist for first and second CPET, with none on beta blockade, $96 \%$ of both LVRS subjects and non-surgical participants were on anticholinergic medications for first and second CPET $(P>.99), 9 \%$ were on digitalis for
Table 1. Baseline Demographics and Pulmonary Function

\begin{tabular}{|c|c|c|c|}
\hline Characteristics & $\begin{array}{c}\text { LVRS } \\
(n=55)\end{array}$ & $\begin{array}{l}\text { Non-surgical } \\
\quad(n=25)\end{array}$ & $P$ \\
\hline Age (y) & $63 \pm 7$ & $65 \pm 7$ & .13 \\
\hline Male gender $(\%)$ & 38 & 64 & $.03 *$ \\
\hline BMI $\left(\mathrm{kg} / \mathrm{m}^{2}\right)$ & $24.4 \pm 4.0$ & $24.6 \pm 3.3$ & .82 \\
\hline $\mathrm{FEV}_{1}(\mathrm{~L})$ & $0.69 \pm 0.18$ & $0.72 \pm 0.25$ & .59 \\
\hline $\mathrm{FEV}_{1}(\%$ pred $)$ & $26 \pm 7$ & $24 \pm 7$ & .11 \\
\hline FVC (\% pred) & $62 \pm 15$ & $58 \pm 16$ & .32 \\
\hline RV (\% pred) & $226 \pm 44$ & $249 \pm 61$ & .06 \\
\hline TLC (\% pred) & $126 \pm 13$ & $132 \pm 15$ & .09 \\
\hline RV/TLC & $0.68 \pm 0.08$ & $0.69 \pm 0.10$ & .71 \\
\hline \multicolumn{4}{|c|}{$\begin{array}{l}\text { Values are given as mean } \pm \mathrm{SD} \text {, unless otherwise indicated. } \\
* \text { Bold } P \text { value indicates significant difference between LVRS and medical group. } \\
\text { LVRS }=\text { lung volume reduction surgery } \\
\text { BMI }=\text { body mass index } \\
\% \text { pred }=\text { percent predicted } \\
\mathrm{RV}=\text { residual volume } \\
\text { TLC }=\text { total lung capacity }\end{array}$} \\
\hline
\end{tabular}

first and second CPET: $8 \%$ of controls and 9\% of LVRS subjects $(P>.99)$, and $24 \%$ were on calcium channel blockers for first and second CPET: $20 \%$ of non-surgical participants and $26 \%$ of LVRS subjects $(P=.60)$.

\section{Maximum Exercise Testing and Ventilatory Efficiency}

At baseline, the non-surgical group had a significantly higher $\dot{\mathrm{V}}_{\mathrm{O}_{2}}(\%$ pred $)$ and peak $\dot{\mathrm{V}}_{\mathrm{E}}$ with a lower breathing reserve than those who received LVRS ( $P$ values $=.04$, .04 , and $<.01$, respectively). All PFTs were comparable at baseline.

From baseline to 6 months, the LVRS group significantly increased their peak $\dot{\mathrm{V}}_{\mathrm{O}_{2}}, \dot{\mathrm{V}}_{\mathrm{O}_{2}}$ (\% pred), work load, peak $\dot{\mathrm{V}}_{\mathrm{E}}$, breathing reserve, peak $\dot{\mathrm{V}}_{\mathrm{CO}_{2}}$, peak $\mathrm{V}_{\mathrm{T}}$, and $\% \mathrm{HRR}$ while lowering their peak $\dot{\mathrm{V}}_{\mathrm{E}} / \dot{\mathrm{V}}_{\mathrm{CO}_{2}}$, lowest $\dot{\mathrm{V}}_{\mathrm{E}} / \dot{\mathrm{V}}_{\mathrm{CO}_{2}}$, and peak $\mathrm{P}_{\mathrm{ETCO}_{2}}$ (Table 2). The non-surgical group only significantly increased their breathing reserve and actually decreased their peak $\dot{\mathrm{V}}_{\mathrm{O}_{2}}, \dot{\mathrm{V}}_{\mathrm{O}_{2}}$ (\% pred), work load, peak $\dot{\mathrm{V}}_{\mathrm{E}}$, and peak $\dot{\mathrm{V}}_{\mathrm{CO}_{2}}$. It is interesting to note, however, that the non-surgical group significantly decreased their $\dot{\mathrm{V}}_{\mathrm{E}} / \dot{\mathrm{V}}_{\mathrm{CO}_{2}}$ intercept. This was only by $0.58 \pm 1.39$, and its relevance as to a clinically meaningful change is questionable.

After 6 months, the LVRS group had a significantly higher work load $(P=.01)$, breathing reserve $(P=.03)$, peak $\dot{\mathrm{V}}_{\mathrm{CO}_{2}}(P<.01)$, and \%HRR $(P<.01)$, compared with the non-surgical group. Additionally, all of the PFTs were significantly better in the LVRS group compared with the non-surgical group (all $P<.001$ ). 
Ventilatory Efficiency Before AND After LVRS

Table 2. Cardiopulmonary Exercise Testing Measurements at Baseline and 6 Months

\begin{tabular}{|c|c|c|c|c|c|c|c|c|}
\hline \multirow{2}{*}{ Characteristics } & \multicolumn{4}{|c|}{ LVRS } & \multicolumn{4}{|c|}{ Non-surgical } \\
\hline & $\begin{array}{l}\text { Baseline } \\
(n=55)\end{array}$ & $\begin{array}{c}6 \text { Mo } \\
(n=55)\end{array}$ & Change & $P$ & $\begin{array}{l}\text { Baseline } \\
(n=25)\end{array}$ & $\begin{array}{c}6 \text { Mo } \\
(n=25) \\
\end{array}$ & Change & $P$ \\
\hline Peak $\dot{\mathrm{V}}_{\mathrm{O}_{2}}(\mathrm{~mL} / \mathrm{min} / \mathrm{kg})$ & $13.6 \pm 3.4$ & $15.7 \pm 3.5$ & $+2.1 \pm 3.1$ & $<.001$ & $15.8 \pm 5.3$ & $14.9 \pm 5.1$ & $-1.0 \pm 2.1$ & .03 \\
\hline Peak $\dot{\mathrm{V}}_{\mathrm{O}_{2}}(\%$ pred $)$ & $48 \pm 14$ & $56 \pm 14$ & $+8 \pm 11$ & $<.001$ & $57 \pm 19$ & $54 \pm 20$ & $-3 \pm 7$ & .05 \\
\hline Peak watts & $38 \pm 15$ & $49 \pm 17$ & $+12 \pm 12$ & $<.001$ & $39 \pm 21$ & $35 \pm 23$ & $-4 \pm 10$ & .04 \\
\hline Peak $\dot{V}_{E}(L / m i n)$ & $24.9 \pm 7.6$ & $31.1 \pm 10.5$ & $+6 \pm 8$ & $<.001$ & $30 \pm 10$ & $28 \pm 10$ & $-2 \pm 4$ & .005 \\
\hline Breathing reserve $(\%)$ & $11 \pm 19$ & $19 \pm 18$ & $+7 \pm 20$ & .01 & $-6 \pm 23$ & $7 \pm 22$ & $+12 \pm 24$ & .01 \\
\hline Peak $\dot{\mathrm{V}}_{\mathrm{CO}_{2}}(\mathrm{~mL} / \mathrm{min} / \mathrm{kg})$ & $11.5 \pm 3.1$ & $14.6 \pm 4.1$ & $+3.2 \pm 3.5$ & $<.001$ & $12.8 \pm 4.6$ & $11.6 \pm 4.1$ & $-1.2 \pm 1.7$ & $<.001$ \\
\hline Peak $\dot{\mathrm{V}}_{\mathrm{E}} / \dot{\mathrm{V}}_{\mathrm{CO}_{2}}$ & $33.3 \pm 5.9$ & $32.1 \pm 4.7$ & $-1.2 \pm 3.5$ & .02 & $34.0 \pm 6.7$ & $34.8 \pm 6.7$ & $+0.8 \pm 3.9$ & .31 \\
\hline Lowest $\dot{\mathrm{V}}_{\mathrm{E}} / \dot{\mathrm{V}}_{\mathrm{CO}_{2}}^{2}$ & $32.5 \pm 5.8$ & $31.5 \pm 4.6$ & $-1.0 \pm 3.4$ & .04 & $33.5 \pm 6.6$ & $34.2 \pm 6.8$ & $+0.6 \pm 4.0$ & .43 \\
\hline$\dot{\mathrm{V}}_{\mathrm{E}} / \dot{\mathrm{V}}_{\mathrm{CO}_{2}}$ slope & $24.6 \pm 5.4$ & $25.2 \pm 5.4$ & $+0.64 \pm 5.5$ & .40 & $26.9 \pm 5.6$ & $27.8 \pm 5.5$ & $+0.9 \pm 5.1$ & .40 \\
\hline$\dot{\mathrm{V}}_{\mathrm{E}} / \dot{\mathrm{V}}_{\mathrm{CO}_{2}}$ intercept & $6.18 \pm 2.33$ & $6.20 \pm 2.56$ & $+0.02 \pm 2.80$ & .96 & $5.83 \pm 2.79$ & $5.25 \pm 2.46$ & $-0.58 \pm 1.39$ & .05 \\
\hline Peak $\mathrm{P}_{\mathrm{ETCO}_{2}}(\mathrm{~mm} \mathrm{Hg})$ & $42.7 \pm 6.3$ & $40.2 \pm 6.3$ & $-2.4 \pm 4.6$ & $<.001$ & $41.5 \pm 7.7$ & $41.2 \pm 7.2$ & $-0.3 \pm 3.6$ & .68 \\
\hline$\% \mathrm{HRR}$ & $42 \pm 15$ & $54 \pm 16$ & $+12 \pm 16$ & $<.001$ & $44 \pm 22$ & $40 \pm 18$ & $-4 \pm 18$ & .26 \\
\hline HR rest (beats/min) & $88 \pm 11$ & $83 \pm 11$ & $-5 \pm 13$ & .01 & $91 \pm 16$ & $93 \pm 15$ & $+2 \pm 8$ & .23 \\
\hline HR peak (beats/min) & $120 \pm 14$ & $126 \pm 15$ & $+7 \pm 14$ & .001 & $121 \pm 20$ & $120 \pm 17$ & $+0.8 \pm 11$ & .70 \\
\hline HR peak (\% pred) & $164.0 \pm 4.7$ & $163.6 \pm 5$ & $-0.5 \pm 0.3$ & $<.001$ & $162.2 \pm 4.7$ & $161.9 \pm 4.7$ & $-0.4 \pm 0.4$ & $<.001$ \\
\hline $\mathrm{O}_{2}$ pulse base (mL/beat) & $3.7 \pm 0.9$ & $4.0 \pm 1.1$ & $+0.3 \pm 1.0$ & .02 & $4.1 \pm 1.4$ & $4.0 \pm 1.4$ & $-0.1 \pm 1.1$ & .53 \\
\hline $\mathrm{O}_{2}$ pulse peak (mL/beat) & $7.6 \pm 2.3$ & $8.5 \pm 2.6$ & $+0.8 \pm 1.6$ & $<.001$ & $9.5 \pm 4.1$ & $9.0 \pm 4.2$ & $-0.5 \pm 1.1$ & .03 \\
\hline Peak $V_{T}(L)$ & $0.88 \pm 0.27$ & $1.15 \pm 0.33$ & $+0.27 \pm 0.24$ & $<.001$ & $0.99 \pm 0.31$ & $0.99 \pm 0.41$ & $-0.0 \pm 0.22$ & .95 \\
\hline $\begin{array}{l}\text { Peak breathing frequency } \\
\text { (breaths } / \mathrm{min} \text { ) }\end{array}$ & $29 \pm 7$ & $28 \pm 6$ & $-1 \pm 5$ & .06 & $31 \pm 8$ & $29 \pm 9$ & $-2 \pm 4$ & .10 \\
\hline \multicolumn{9}{|c|}{$\begin{array}{l}\text { Values are given as mean } \pm \mathrm{SD} \text {. } \\
\mathrm{V}_{\mathrm{O}_{2}}=\text { volume of oxygen consumed } \\
\mathrm{V}_{\mathrm{E}}=\text { minute ventilation } \\
\dot{\mathrm{V}}_{\mathrm{CO}_{2}}=\text { rate of carbon dioxide produced } \\
\mathrm{P}_{\mathrm{ETCO}}=\text { partial pressure of end-tidal carbon dioxide } \\
\% \mathrm{HRR}=\text { percent predicted heart rate reserve } \\
\mathrm{HR}=\text { heart rate } \\
\% \text { pred }=\text { percent predicted } \\
\mathrm{V}_{\mathrm{T}}=\text { tidal volume }\end{array}$} \\
\hline
\end{tabular}

\section{LVRS Subjects Who Significantly Improved Exercise Capacity}

We then compared the LVRS subjects who significantly improved their exercise capacity $(>10 \mathrm{~W})$ after surgery to those who did not $(\leq 10 \mathrm{~W}$ ) (Table 3$)$. The only significant baseline difference between these two groups was that those who improved had lower watts $(P=.04)$. Six months after surgery, those who improved significantly increased their $\mathrm{BMI}, \mathrm{FEV}_{1}(\%$ pred $), \mathrm{FVC}(\%$ pred $)$, peak $\dot{\mathrm{V}}_{\mathrm{O}_{2}}, \dot{\mathrm{V}}_{\mathrm{O}_{2}}(\%$ pred $)$, peak watts, peak $\dot{\mathrm{V}}_{\mathrm{E}}$, breathing reserve, peak $\dot{\mathrm{V}}_{\mathrm{CO}_{2}}$, peak $\mathrm{V}_{\mathrm{T}}$, and \%HRR while decreasing their $\mathrm{RV}(\%$ pred $), \mathrm{TLC}(\%$ pred $), \mathrm{RV} / \mathrm{TLC}$, peak $\dot{\mathrm{V}}_{\mathrm{E}} / \dot{\mathrm{V}}_{\mathrm{CO}_{2}}$, lowest $\dot{\mathrm{V}}_{\mathrm{E}} / \dot{\mathrm{V}}_{\mathrm{CO}_{2}}$ (Fig. 2), and peak $\mathrm{P}_{\mathrm{ETCO}_{2}}$. Those who did not improve failed to increase their peak $\dot{\mathrm{V}}_{\mathrm{O}_{2}}, \dot{\mathrm{V}}_{\mathrm{O}_{2}}(\%$ pred $)$, peak watts, breathing reserve, while also failing to decrease their peak $\dot{\mathrm{V}}_{\mathrm{E}} / \dot{\mathrm{V}}_{\mathrm{CO}_{2}}$ and lowest $\dot{\mathrm{V}}_{\mathrm{E}} / \dot{\mathrm{V}}_{\mathrm{CO}_{2}}$. Additionally, the correlation between the change in watts and the change in $\dot{\mathrm{V}}_{\mathrm{E}} / \dot{\mathrm{V}}_{\mathrm{CO}_{2}}$ was $\mathrm{r}=-0.288$ with a $P$ value $=.033$.

The 26 subjects who did improve their exercise capacity (improvers) had significantly better $\operatorname{FEV}_{1}(P<.01)$,
$\mathrm{FEV}_{1}(\%$ pred $)(P=.001), \mathrm{FVC}(\%$ pred $)(P<.01), \mathrm{RV}(\%$ pred) $(P=.01)$, and RV/TLC $(P=.01)$ at 6-month testing compared with the 29 subjects who did not improve exercise capacity (non-improvers). Additionally, improvers had significantly higher $\dot{\mathrm{V}}_{\mathrm{O}_{2}}(\%$ pred $)(P=.03)$, work load $(P=.005)$, peak $\dot{\mathrm{V}}_{\mathrm{E}}(P=.04)$, and breathing reserve $(P=.04)$; actual values are found in Table 3 .

\section{Iso-Work Load Comparisons}

We then collected CPET measurements at the same work load before and after LVRS. Responses to identical levels of exercise before and after LVRS for the overall group and those who improved and did not improve their exercise capacity are shown in Table 4 . Only 1 non-surgical participant significantly improved exercise capacity, and none of the variables significantly changed in the overall non-surgical group, in the single non-surgical participant who improved, or in non-surgical participants who did not improve at iso-work load (data not shown). For the LVRS subjects (Table 4), all 3 groups (overall, improvers, and 


\section{Ventilatory Efficiency Before and After LVRS}

Table 3. Changes After LVRS: Subjects Who Significantly Improved Their Workload and Subjects Who Did Not

\begin{tabular}{|c|c|c|c|c|c|c|}
\hline \multirow{2}{*}{ Characteristics } & \multicolumn{3}{|c|}{$>10 \mathrm{~W}$ Improvement } & \multicolumn{3}{|c|}{$\leq 10 \mathrm{~W}$ Improvement } \\
\hline & $\begin{array}{l}\text { Baseline } \\
(n=26)\end{array}$ & $\begin{array}{c}6 \text { Mo } \\
(n=26)\end{array}$ & $P$ & $\begin{array}{l}\text { Baseline } \\
(n=29)\end{array}$ & $\begin{array}{c}6 \text { Mo } \\
(n=29)\end{array}$ & $P$ \\
\hline Age (y) & $63 \pm 6$ & $64 \pm 6$ & & $62 \pm 8$ & $63 \pm 8$ & \\
\hline Male gender (\%) & 39 & & & 38 & & \\
\hline BMI, $\mathrm{kg} / \mathrm{m}^{2}$ & $24.9 \pm 4.5$ & $25.4 \pm 4.3$ & .09 & $23.9 \pm 3.5$ & $24.0 \pm 4.0$ & .80 \\
\hline $\mathrm{FEV}_{1}(\mathrm{~L})$ & $0.68 \pm 0.17$ & $1.14 \pm 0.37$ & $<.001$ & $0.76 \pm 0.33$ & $0.87 \pm 0.27$ & .13 \\
\hline $\mathrm{FEV}_{1}(\%$ pred $)$ & $26 \pm 6$ & $45 \pm 14$ & $<.001$ & $26 \pm 8$ & $33 \pm 11$ & $<.001$ \\
\hline FVC (\% pred) & $61 \pm 13$ & $88 \pm 15$ & $<.001$ & $62 \pm 17$ & $76 \pm 14$ & $<.001$ \\
\hline RV (\% pred) & $227 \pm 39$ & $149 \pm 38$ & $<.001$ & $225 \pm 48$ & $172 \pm 43$ & $<.001$ \\
\hline TLC (\% pred) & $128 \pm 13$ & $113 \pm 15$ & $<.001$ & $125 \pm 14$ & $114 \pm 16$ & $<.001$ \\
\hline RV/TLC & $0.69 \pm 0.07$ & $0.50 \pm 0.09$ & $<.001$ & $0.68 \pm 0.08$ & $0.56 \pm 0.08$ & $<.001$ \\
\hline Peak $\dot{\mathrm{V}}_{\mathrm{O}_{2}}(\mathrm{~mL} / \mathrm{min} / \mathrm{kg})$ & $12.8 \pm 3.7$ & $16.6 \pm 3.2$ & $<.001$ & $14.3 \pm 2.9$ & $14.9 \pm 3.7$ & .22 \\
\hline$\dot{\mathrm{V}}_{\mathrm{O}_{2}}(\%$ pred $)$ & $46 \pm 16$ & $61 \pm 16$ & $<.001$ & $51 \pm 12$ & $52 \pm 12$ & .30 \\
\hline Peak watts & $34 \pm 18$ & $56 \pm 20$ & $<.001$ & $41 \pm 11$ & $43 \pm 11$ & .14 \\
\hline Peak $\dot{V}_{\mathrm{E}}(\mathrm{L} / \mathrm{min})$ & $24.0 \pm 8.6$ & $34.3 \pm 12.7$ & $<.001$ & $25.7 \pm 6.8$ & $28.3 \pm 7.2$ & .02 \\
\hline Breathing reserve (\%) & $15 \pm 18$ & $24 \pm 17$ & .03 & $8 \pm 20$ & $14 \pm 18$ & .15 \\
\hline Peak $\dot{\mathrm{V}}_{\mathrm{CO}_{2}}(\mathrm{~mL} / \mathrm{min} / \mathrm{kg})$ & $10.6 \pm 3.3$ & $15.7 \pm 4.0$ & $<.001$ & $12.3 \pm 2.9$ & $13.7 \pm 4.0$ & .01 \\
\hline Peak $\dot{\mathrm{V}}_{\mathrm{E}} / \dot{\mathrm{V}}_{\mathrm{CO}_{2}}$ & $34.4 \pm 5.9$ & $32.0 \pm 4.8$ & $<.001$ & $32.3 \pm 5.8$ & $32.2 \pm 4.8$ & .94 \\
\hline Lowest $\dot{\mathrm{V}}_{\mathrm{E}} / \dot{\mathrm{V}}_{\mathrm{CO}_{2}}$ & $33.5 \pm 5.5$ & $31.5 \pm 4.6$ & $<.001$ & $31.6 \pm 6.0$ & $31.6 \pm 6.0$ & .93 \\
\hline$\dot{\mathrm{V}}_{\mathrm{E}} / \dot{\mathrm{V}}_{\mathrm{CO}_{2}}$ slope & $25.1 \pm 6.2$ & $25.9 \pm 5.8$ & .54 & $24.0 \pm 4.7$ & $24.5 \pm 5.0$ & .55 \\
\hline$\dot{\mathrm{V}}_{\mathrm{E}} / \dot{\mathrm{V}}_{\mathrm{CO}_{2}}$ intercept & $6.15 \pm 2.31$ & $6.28 \pm 3.06$ & .86 & $6.20 \pm 2.39$ & $6.12 \pm 2.07$ & .83 \\
\hline Peak $\mathrm{P}_{\mathrm{ETCO}_{2}}(\mathrm{~mm} \mathrm{Hg})$ & $41.9 \pm 5.1$ & $39.5 \pm 6.1$ & .006 & $43.9 \pm 7.8$ & $40.9 \pm 6.5$ & .02 \\
\hline$\% \mathrm{HRR}$ & $41 \pm 16$ & $59 \pm 17$ & $<.001$ & $44 \pm 15$ & $50 \pm 16$ & .04 \\
\hline HR rest (beats/min) & $86 \pm 9$ & $83 \pm 13$ & .24 & $89 \pm 13$ & $83 \pm 9$ & .01 \\
\hline HR peak (beats/min) & $117 \pm 14$ & $129 \pm 16$ & $<.001$ & $122 \pm 14$ & $124 \pm 15$ & .37 \\
\hline HR peak (\% pred) & $163.7 \pm 3.9$ & $163.3 \pm 3.8$ & $<.001$ & $164.3 \pm 5.4$ & $163.9 \pm 5.3$ & $<.001$ \\
\hline $\mathrm{O}_{2}$ pulse base (mL/beat) & $3.7 \pm 0.9$ & $4.1 \pm 1.1$ & .07 & $3.7 \pm 0.8$ & $4.0 \pm 1.1$ & .13 \\
\hline $\mathrm{O}_{2}$ pulse peak (mL/beat) & $7.4 \pm 2.8$ & $9.0 \pm 2.9$ & $<.001$ & $7.9 \pm 1.9$ & $7.9 \pm 2.1$ & .76 \\
\hline Peak $V_{T}(L)$ & $0.84 \pm 0.26$ & $1.22 \pm 0.36$ & $<.001$ & $0.92 \pm 0.27$ & $1.08 \pm 0.30$ & $<.001$ \\
\hline $\begin{array}{l}\text { Peak breathing frequency } \\
\text { (breaths/min) }\end{array}$ & $29 \pm 8$ & $28 \pm 6$ & .655 & $29 \pm 7$ & $27 \pm 8$ & .02 \\
\hline $\begin{array}{l}\text { Values are given as mean } \pm \mathrm{SD} \text {, un } \\
\mathrm{LVRS}=\text { lung volume reduction sur } \\
\mathrm{BMI}=\text { body mass index } \\
\mathrm{RV}=\text { residual volume } \\
\mathrm{TLC}=\text { total lung capacity } \\
\mathrm{V}_{\mathrm{O}_{2}}=\text { volume of oxygen consumec } \\
\mathrm{V}_{\mathrm{E}}=\text { minute ventilation } \\
\mathrm{V}_{\mathrm{CO}_{2}}=\text { rate of carbon dioxide prod } \\
\mathrm{P}_{\mathrm{ETCO}}=\text { partial pressure of end-tic } \\
\% \mathrm{HRR}=\text { percent predicted heart ra } \\
\mathrm{HR}=\text { heart rate } \\
\% \text { pred = percent predicted } \\
\mathrm{V}_{\mathrm{T}}=\text { tidal volume }\end{array}$ & wise indicated. & & & & & \\
\hline
\end{tabular}

non-improvers) increased their $\mathrm{V}_{\mathrm{T}}$ significantly. The improved work load group also significantly increased their respiratory exchange ratio while decreasing their breathing frequency and $\mathrm{P}_{\mathrm{ETCO}_{2}}$.

\section{Discussion}

In support of our hypothesis, we found that compared with the non-surgical group, LVRS subjects had improved ventilatory efficiency as measured by a small but signifi- cant decrease in their peak $\dot{\mathrm{V}}_{\mathrm{E}} / \dot{\mathrm{V}}_{\mathrm{CO}_{2}}$ and lowest $\dot{\mathrm{V}}_{\mathrm{E}} / \dot{\mathrm{V}}_{\mathrm{CO}_{2}}$ from baseline to 6 months. The changes were greatest in the LVRS subjects who showed the most functional improvement measured by peak wattage, indicating an association of improved ventilation with improved functional outcome as measured by CPET. This is supported by the fact that those who significantly improved their wattage also increased their $\dot{\mathrm{V}}_{\mathrm{E}}$ significantly more than those who did not improve.

The severity of COPD is assessed using resting PFTs, but this does not usually correspond to exercise impair- 


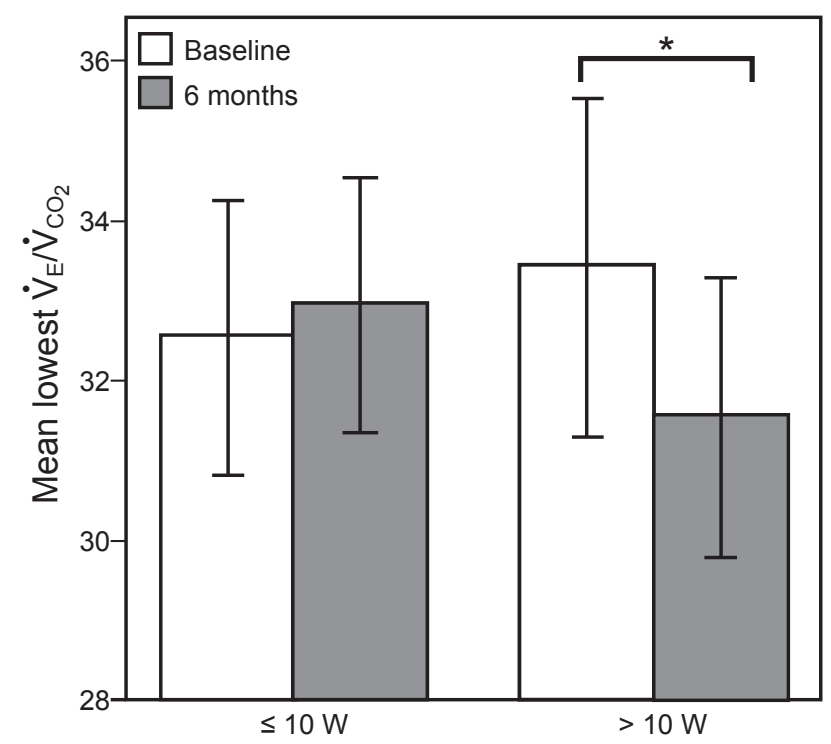

Fig. 2. Bar graphs show that lung volume reduction surgery subjects who significantly improved their exercise capacity $(>10 \mathrm{~W})$ also significantly improved their ventilatory efficiency (peak $\dot{\mathrm{V}}_{\mathrm{E}} / \dot{\mathrm{V}}_{\mathrm{CO}_{2}}$ ) compared to those who did not improve their exercise capacity. Data are shown as mean $\pm \mathrm{SD}$. ${ }^{*} P \leq .05$.

ment in individual patients. ${ }^{23}$ Although multifactorial, one of the biggest reasons for exercise intolerance in COPD is the inability to increase $\dot{V}_{\mathrm{E}}$ relative to higher work loads with higher end-expiratory volumes and air trapping. Ventilatory limitation is typically classified by a breathing reserve $<15 \% .{ }^{23,36}$ In patients with COPD, this results from a reduction in MVV, increased dead space ventilation, and the inefficiency of gas exchange, raising the required $\dot{V}_{\mathrm{E}}$ at any metabolic rate. ${ }^{23}$ After LVRS, the elastic recoil increases, ${ }^{28,40}$ thereby decreasing TLC and RV and decreasing hyperinflation, which could explain the increase in peak $\dot{\mathrm{V}}_{\mathrm{E}}$ seen in our subjects. Criner et $\mathrm{al}^{26}$ found in their NETT exercise substudy that the improvements in exercise capacity following LVRS were due to less rapid and shallow breathing, reductions in ventilatory dead space, and enhanced carbon dioxide elimination. Our results support these findings, as those who improved their exercise capacity also significantly increased their peak $\dot{\mathrm{V}}_{\mathrm{E}}$ and $\dot{\mathrm{V}}_{\mathrm{CO}_{2}}$. In the subjects who improved their exercise capacity, the RV/TLC ratio was significantly lower, demonstrating less air trapping which leads to more efficient ventilation. Additionally, those who improved their exercise capacity showed a small but significant decrease in their peak $\dot{\mathrm{V}}_{\mathrm{E}} / \dot{\mathrm{V}}_{\mathrm{CO}_{2}}$ and lowest $\dot{\mathrm{V}}_{\mathrm{E}} / \dot{\mathrm{V}}_{\mathrm{CO}_{2}}$.

Patients with emphysema have an increased physiological dead space to tidal volume ratio $\left(\mathrm{V}_{\mathrm{D}} / \mathrm{V}_{\mathrm{T}}\right)$ that fails to decrease normally during exercise ${ }^{41,42}$ and contributes to respiratory acidemia and resulting exercise limitation. ${ }^{27}$ Benditt and colleagues ${ }^{27}$ found, for their group of LVRS subjects, that the $\mathrm{V}_{\mathrm{D}} / \mathrm{VT}$ decreased slightly at rest and noticeably during exercise. The authors noted that, although a reduction in $V_{D} / V_{T}$ should improve ventilatory efficiency and decrease the $\dot{V}_{\mathrm{E}}$ needed for a given work load, they saw no change in the $\dot{\mathrm{V}}_{\mathrm{E}}$ measured at the point of isowatt exercise. This is most likely because they found the partial

Table 4. Comparisons of Metabolic and Ventilatory Variables During Iso-Work Load Exercise

\begin{tabular}{|c|c|c|c|c|c|c|}
\hline Characteristics & $\begin{array}{c}\text { Total } \\
\text { LVRS } \\
\text { Baseline } \\
(n=55)\end{array}$ & $\begin{array}{c}\text { Total } \\
\text { LVRS } \\
6 \text { Mo } \\
(n=55)\end{array}$ & $\begin{array}{c}>10 \mathrm{~W} \\
\text { Improvement } \\
\text { Baseline } \\
(n=26)\end{array}$ & $\begin{array}{c}>10 \mathrm{~W} \\
\text { Improvement } \\
6 \mathrm{Mo} \\
(n=26)\end{array}$ & $\begin{array}{c}\leq 10 \mathrm{~W} \\
\text { Improvement } \\
\text { Baseline } \\
(n=29)\end{array}$ & $\begin{array}{c}\leq 10 \mathrm{~W} \\
\text { Improvement } \\
6 \mathrm{Mo} \\
(n=29)\end{array}$ \\
\hline Iso-work load* & $37 \pm 15$ & $37 \pm 15$ & $34 \pm 18$ & $34 \pm 19$ & $39 \pm 10$ & $39 \pm 10$ \\
\hline$\dot{\mathrm{V}}_{\mathrm{E}}(\mathrm{L} / \mathrm{min})$ & $24.5 \pm 7.6$ & $26.3 \pm 8.1 \dagger$ & $24.0 \pm 8.6$ & $26.6 \pm 9.7$ & $25.0 \pm 6.8$ & $26.8 \pm 6.5$ \\
\hline$\dot{\mathrm{V}}_{\mathrm{CO}_{2}}(\mathrm{~mL} / \mathrm{min} / \mathrm{kg})$ & $11.2 \pm 3.2$ & $11.9 \pm 3.5 \dagger$ & $10.6 \pm 3.2$ & $11.0 \pm 3.2$ & $11.8 \pm 3.2$ & $12.7 \pm 3.8$ \\
\hline$\dot{\mathrm{V}}_{\mathrm{E}} / \dot{\mathrm{V}}_{\mathrm{CO}_{2}}$ & $34 \pm 6$ & $34 \pm 5$ & $34 \pm 6$ & $35 \pm 5$ & $33 \pm 6$ & $33 \pm 5$ \\
\hline $\mathrm{P}_{\mathrm{ETCO}_{2}}(\mathrm{~mm} \mathrm{Hg})$ & $42.5 \pm 6.4$ & $38.8 \pm 6.3 \ddagger$ & $41.9 \pm 5.1$ & $37.7 \pm 5.1 \neq$ & $43.0 \pm 7.4$ & $39.8 \pm 7.2$ \\
\hline$\dot{\mathrm{V}}_{\mathrm{O}_{2}}(\mathrm{~mL} / \mathrm{min} / \mathrm{kg})$ & $13.3 \pm 3.5$ & $13.1 \pm 3.8$ & $12.8 \pm 3.7$ & $12.4 \pm 3.3$ & $13.7 \pm 3.2$ & $13.7 \pm 4.2$ \\
\hline $\mathrm{V}_{\mathrm{T}}(\mathrm{L})$ & $0.88 \pm 0.26$ & $1.14 \pm 0.37 \dagger$ & $0.84 \pm 0.26$ & $1.21 \pm 0.40 \dagger$ & $0.91 \pm 0.25$ & $1.08 \pm 0.33 \dagger$ \\
\hline RER & $0.84 \pm 0.11$ & $1.00 \pm 0.81$ & $0.83 \pm 0.12$ & $0.88 \pm 0.07 \dagger$ & $0.85 \pm 0.10$ & $1.11 \pm 1.12$ \\
\hline Breathing frequency (breaths/min) & $29 \pm 8$ & $26 \pm 13$ & $29 \pm 8$ & $23 \pm 11 \ddagger$ & $29 \pm 8$ & $28 \pm 15$ \\
\hline $\begin{array}{l}\text { Values are given as mean } \pm \mathrm{SD} \text {, unless otheru } \\
* \text { Iso-work load: same wattage before and afte } \\
\dagger \text { Significant increase }(P \leq .05) \text { from baseline } \\
\text { ‡ Significant decrease }(P \leq .05) \text { from baseline } \\
\mathrm{LVRS}=\text { lung volume reduction surgery } \\
\dot{\mathrm{V}}_{\mathrm{E}}=\text { minute ventilation } \\
\dot{\mathrm{V}}_{\mathrm{CO}_{2}}=\text { rate of carbon dioxide produced } \\
\mathrm{P}_{\mathrm{ETCO}}=\text { partial pressure of end-tidal carbon } \\
\dot{\mathrm{V}}_{\mathrm{O}_{2}}=\text { volume of oxygen consumed } \\
\mathrm{V}_{\mathrm{T}}=\text { tidal volume } \\
\text { RER = respiratory exchange ratio }\end{array}$ & $\begin{array}{l}\text { dicated. } \\
\text { S. } \\
\text { no. } \\
\text { no. }\end{array}$ & & & & & \\
\hline
\end{tabular}


pressure of arterial carbon dioxide to be lower at the isowatt level after surgery due to the increased alveolar ventilation. ${ }^{27}$ Another study by Criner et $\mathrm{al}^{26}$ showed that there was a decrease in ventilatory dead space during unloaded cycling and at maximum exercise after LVRS. With the increase in peak $\dot{V}_{E}$, the reduced dead space also suggested improvements in alveolar ventilation throughout exercise that resulted in improved carbon dioxide elimination. Similarly, in our LVRS group at identical work loads during the ramped exercise test (iso-work load) and at peak exercise, there was an increase in $\dot{\mathrm{V}}_{\mathrm{E}}$. This was coupled with a decrease in $\mathrm{P}_{\mathrm{ETCO}_{2}}$. This may help to explain why the change in $\dot{\mathrm{V}}_{\mathrm{E}} / \dot{\mathrm{V}}_{\mathrm{CO}_{2}}$ was small. Because the $\mathrm{P}_{\mathrm{ETCO}_{2}}$ values were lower, this suggests a lower alveolar $\mathrm{P}_{\mathrm{CO}_{2}}$. Because our subjects did not increase their $\dot{\mathrm{V}}_{\mathrm{E}} / \mathrm{V}_{\mathrm{CO}_{2}}$, despite a lower alveolar $\mathrm{P}_{\mathrm{CO}_{2}}$, one can infer that their V/Q distribution improved. Although $\dot{\mathrm{V}}_{\mathrm{E}} / \dot{\mathrm{V}}_{\mathrm{CO}_{2}}$ reflects the total breathing effort required to eliminate carbon dioxide, improved ventilatory function can cause it to go either up or down.

Our findings are in agreement with those of Criner et al, ${ }^{26}$ who found that the improvement in ventilatory function (improved peak $\dot{\mathrm{V}}_{\mathrm{E}}$, reduced dead space, and improved carbon dioxide elimination though alveolar ventilation) is the major benefit of LVRS in terms of exercise performance. In our study, those who significantly increased their work load also significantly improved their peak $\dot{V}_{E}$, peak $\dot{\mathrm{V}}_{\mathrm{CO}_{2}}$, peak $\mathrm{V}_{\mathrm{T}}$, breathing reserve, peak $\dot{\mathrm{V}}_{\mathrm{E}} / \dot{\mathrm{V}}_{\mathrm{CO}_{2}}$, and lowest $\dot{\mathrm{V}}_{\mathrm{E}} / \dot{\mathrm{V}}_{\mathrm{CO}_{2}}$. Those who did not increase their work load did not improve these variables, or at least not to the same degree. In the study by Criner et al, ${ }^{26} 15 \%$ of the subjects improved their exercise capacity by $>10 \mathrm{~W}$; however, in our study, $47 \%$ significantly improved their exercise capacity. This is most likely attributed to better selection criteria after the NETT results. The differences between those who improved their exercise capacity and those who did not are most likely due to better pulmonary function outcomes. Those who improved had significantly better $\mathrm{FEV}_{1}, \mathrm{FEV}_{1}(\%$ pred $), \mathrm{FVC}(\%$ pred $), \mathrm{RV}(\%$ pred $)$, and RV/TLC at 6-month testing compared with those who did not improve their exercise capacity.

In a study measuring ventilatory efficiency in 474 adults, Sun and colleagues ${ }^{24}$ showed that the lowest $\dot{\mathrm{V}}_{\mathrm{E}} / \dot{\mathrm{V}}_{\mathrm{CO}_{2}}$ ratio was the most reproducible and stable response accounting for laboratory sites, exercise mode, gender, and age. It has been hypothesized that the $\dot{\mathrm{V}}_{\mathrm{E}} / \dot{\mathrm{V}}_{\mathrm{CO}_{2}}$ ratio may be a more stable marker of ventilatory efficiency, as the slope is subject to transient hyperventilation early in exercise and can be affected by metabolic acidosis. ${ }^{24,25}$ This supports the use in our study of peak and lowest $\dot{\mathrm{V}}_{\mathrm{E}} / \dot{\mathrm{V}}_{\mathrm{CO}_{2}}$.

Study limitations include the facts that non-surgical and LVRS groups were not perfectly matched and that data were collected retrospectively. Additionally, the non-surgical subject group was not temporally identical to all of the surgery subjects because it is not ethical to delay LVRS to eligible candidates. Finally, most of the subjects assessed after the NETT had video-assisted thoracoscopic surgery, whereas the subjects in the NETT period all had median sternotomy. However, there has not been any indication in published studies that the surgical approach affects long-term functional outcomes. ${ }^{43}$ Strengths of this study are that it has a relatively large group of unique subjects with data collected on both types of surgeries in a single NETT center with a single exercise lab and using the NETT protocols and rehabilitation guidelines throughout.

\section{Conclusions}

These study results show that LVRS improves ventilatory efficiency, as measured by the peak $\dot{\mathrm{V}}_{\mathrm{E}} / \mathrm{V}_{\mathrm{CO}_{2}}$ and lowest $\dot{\mathrm{V}}_{\mathrm{E}} / \dot{\mathrm{V}}_{\mathrm{CO}_{2}}$ ratio on CPET. It is further demonstrated that the greatest improvement is seen in those who increase their peak wattage by greater than $10 \mathrm{~W}$ from baseline to 6 months after LVRS. The change in $\dot{\mathrm{V}}_{\mathrm{E}} / \dot{\mathrm{V}}_{\mathrm{CO}_{2}}$, although significant, is most likely a small change that agrees with the decrease in $\mathrm{P}_{\mathrm{ETCO}_{2}}$, which suggests an improvement in alveolar ventilation during exercise and results in improved carbon dioxide elimination. Future investigations are needed to determine whether the change in $\dot{\mathrm{V}}_{\mathrm{E}} / \dot{\mathrm{V}}_{\mathrm{CO}_{2}}$ is associated with the known quality of life, dyspnea, morbidity, or mortality benefits of LVRS.

\section{REFERENCES}

1. Corrà U, Mezzani A, Bosimini E, Scapellato F, Temporelli PL, Eleuteri E, Giannuzzi P. Limited predictive value of cardiopulmonary exercise indices in patients with moderate chronic heart failure treated with carvedilol. Am Heart J 2004;147(3):553-560.

2. Brunelli A, Belardinelli R, Pompili C, Xiumé F, Refai M, Salati M, Sabbatini A. Minute ventilation-to-carbon dioxide output (VE/VCO2) slope is the strongest predictor of respiratory complications and death after pulmonary resection. Ann Thoracic Surg 2012;93(6):1802-1806.

3. Torchio R, Guglielmo M, Giardino R, Ardissone F, Ciacco C, Gulotta $\mathrm{C}$, et al. Exercise ventilatory inefficiency and mortality in patients with chronic obstructive pulmonary disease undergoing surgery for non-small-cell lung cancer. Eur J Cardiothorac Surg 2010; 38(1):14-19.

4. Arena R. Peak VO2 and VE/VCO2 slope in patients with heart failure: a prognostic comparison. Am Heart J 2004;147(2):354-360.

5. Bard RL. Determining the best ventilatory efficiency measure to predict mortality in patients with heart failure. J Heart Lung Transplant 2006;25(5):589-595.

6. Nanas SN. VE/VCO2 slope is associated with abnormal resting haemodynamics and is a predictor of long-term survival in chronic heart failure. Eur J Heart Fail 2006;8(4):420-427.

7. Arena R. The ventilatory classification system effectively predicts hospitalization in patients with heart failure. J Cardiopulm Rehabil Prev 2008;28(3):195-198.

8. Mejhert M, Linder-Klingsell E, Edner M, Kahan T, Persson H. Ventilatory variables are strong prognostic markers in elderly patients with heart failure. Heart 2002;88(3):239-243. 


\section{Ventilatory EfFiciency Before and After LVRS}

9. Caviedes IR, Delgado I, Soto R. Ventilatory inefficiency as a limiting factor for exercise in patients with COPD. Respir Care 2012; 57(4):583-589.

10. Teopompi E, Tzani P, Aiello M, Ramponi S, Visca D, Gioia MR, et al. Ventilatory response to carbon dioxide output in patients with chronic heart failure and in patients with COPD with comparable exercise capacity. Respir Care 2014;59(7):1034-1041.

11. Thirapatarapong W, Armstrong HF, Thomashow BM, Bartels MN. Differences in gas exchange between severities of chronic obstructive pulmonary disease. Respir Physiol Neurobiol 2013;186(1):81-86.

12. Agostoni P, Apostolo A, Sciomer S. Evolution of the concept of ventilatory limitation during exercise: combining the pneumologist and cardiologist point of view. Respir Physiol Neurobiol 179(2-3): 127-128, 2011

13. Fishman A, Martinez F, Naunheim K, Piantadosi S, Wise R, Ries A, et al. A randomized trial comparing lung volume reduction surgery with medical therapy for severe emphysema. N Engl J Med 2003; 348(21):2059-2073

14. Minino AM, Xu J, Kochanek KD. Deaths: preliminary data for 2008. Natl Vital Stat Rep 2010;59(2).

15. World Health Organization. World health statistics. France: WHO Press; 2008

16. Serna DL, Brenner M, Osann KE, McKenna RJ Jr, Chen JC, Fischel $\mathrm{RJ}$, et al. Survival after unilateral versus bilateral lung volume reduction surgery for emphysema J Thorac Cardiovasc Surg 1999;118: 1101-1109.

17. Martinez FJ, de Oca MM, Whyte RI, Stetz J, Gay SE, Celli BR. Lung-volume reduction improves dyspnea, dynamic hyperinflation, and respiratory muscle function. Am J Respir Crit Care Med 1997; 155(6):1984-1990.

18. Imfeld S, Bloch KE, Weder W, Russi EW. The BODE index after lung volume reduction surgery correlates with survival. Chest J 2006; 129(4):873-878

19. Stirling GR, Babidge WJ, Peacock MJ, Smith JA, Matar KS, Snell GI, et al. Lung volume reduction surgery in emphysema: a systematic review. Ann Thorac Surg 2001;72(2):641-648.

20. Flaherty KR, Kazerooni EA, Curtis JL, Iannettoni M, Lange L, Schork MA, Martinez FJ. Short-term and long-term outcomes after bilateral lung volume reduction surgery. Chest 2001;119(5):1337-1346.

21. Ferguson GT, Fernandez E, Zamora MR, Pomerantz M, Buchholz J, Make BJ. Improved exercise performance following lung volume reduction surgery for emphysema. Am J Respir Crit Care Med 1998; 157(4 Pt 1):1195-1203.

22. Dolmage TE, Waddell TK, Maltais F, Guyatt GH, Todd TRJ, Keshavjee $\mathrm{S}$, et al. The influence of lung volume reduction surgery on exercise in patients with COPD. Eur Respir J 2004;23:269-274.

23. Arena R, Sietsema KE. Cardiopulmonary exercise testing in the clinical evaluation of patients with heart and lung disease. Circulation 2011;123(6):668-680.

24. Sun XG, Hansen JE, Garatachea N, Storer TW, Wasserman K. Ventilatory Efficiency during exercise in healthy subjects. Am J Respir Crit Care Med 2002;166(11):1443-1448.

25. Myers J, Arena R, Oliveira RB, Bensimhon D, Hsu L, Chase P, et al. The lowest VE/VCO2 ratio during exercise as a predictor of outcomes in patients with heart failure. J Card Fail 2009;15(9):756-762.

26. Criner GJ, Belt P, Sternberg AL, Mosenifar Z, Make BJ, Utz JP, et al. Effects of lung volume reduction surgery on gas exchange and breathing pattern during maximum exercise. Chest J 2009;135(5): 1268-1279.
27. Benditt JO, Lewis S, Wood DE, Klima L, Albert RK. Lung volume reduction surgery improves maximal $\mathrm{O}_{2}$ consumption, maximal minute ventilation, $\mathrm{O}_{2}$ pulse, and dead space-to-tidal volume ratio during leg cycle ergometry. Am J Respir Crit Care Med 1997;156(2):561566.

28. Sciurba FC, Rogers RM, Keenan RJ, Slivka WA, Gorcsan J 3rd, Ferson PF, et al. Improvement in pulmonary function and elastic recoil after lung-reduction surgery for diffuse emphysema. N Engl J Med 1996;334(17):1095-1099.

29. Kim V, Kretschman DM, Sternberg AL, DeCamp MM Jr, Criner GJ, National Emphysema Treatment Trial Research Group. Weight gain after lung reduction surgery is related to improved lung function and ventilatory efficiency. Am J Respir Crit Care Med 2012;186(11): 1109-1116

30. Oswald-Mammosser O, Kessler R, Massard G, Wihlm JM, Weitzenblum E, Lonsdorfer J. Effect of lung volume reduction surgery on gas exchange and pulmonary hemodynamics at rest and during exercise. Am J Respir Crit Care Med 1998;158(4):1020-1025.

31. Centers for Medicare and Medicaid Coverage. Medicare national coverage (NCD) determinations manual. http://www.cms.gov/ Regulations-and-Guidance/Guidance/Manuals/Internet-OnlyManuals-IOMs.html. Accessed September 25, 2014.

32. Ries AL, Make BJ, Lee SM, Krasna MJ, Bartels M, Crouch R, et al. The effects of pulmonary rehabilitation in the national emphysema treatment trial. Chest J 2005;128(6):3799-3809.

33. Crapo RO, Morris AH. Standardized single-breath normal values for carbon monoxide diffusing capacity. Am Rev Respir Dis 1981;123(2): 185-189.

34. Crapo R, Morris A, Clayton P, Nixon C. Lung volumes in healthy nonsmoking adults. Bull Europ Physiopathol Respir 1982;18:419425 .

35. Crapo R, Morris A, Gardner R. Reference spirometric values using techniques and equipment that meet ATS recommendations. Am Rev Respir Dis 1981;123(6):659-664.

36. American Thoracic Society, American College of Chest Physicians. ATS/ACCP statement on cardiopulmonary exercise testing. Am J Respir Crit Care Med 2003;167(2):211-277.

37. Wasserman K, Hansen J, Sue D, Stringer W, Whipp B. Principles of exercise testing and interpretation, 3rd edition. Philadelphia: Lippincott Williams \& Wilkins; 1999.

38. Tanaka H, Monahan K, Seals D. Age-predicted maximal heart rate revisited. J Am Coll Cardiol 2001;37(1):15-16.

39. Lauer MS, Francis GS, Okin PM, Pashkow FJ, Snader CE, Marwick TH. Impaired chronotopic response to exercise stress testing as a predictor of mortality. JAMA 1999;281(6).

40. Gelb AF, McKenna JRJ, Brenner M, Fischel R, Baydur A, Zamel N. Contribution of lung and chest wall mechanics following emphysema resection. Chest J 1996;110(1):11-17.

41. Nery LE, Wasserman K, French W, Oren A, Davis JA. Contrasting cardiovascular and respiratory responses to exercise in mitral valve and chronic obstructive pulmonary diseases. Chest J 1983;83(3): 446-453.

42. Spiro S, Hahn H, Edwards RH, Pride NB. An analysis of the physiological strain of submaximal exercise in patients with chronic obstructive bronchitis. Thorax 1975;30(4):415-425.

43. Huang W, Wang W, Deng B, Tan Y, Jiang G, Zhou H, et al. Several clinical interests regarding lung volume reduction surgery for severe emphysema: meta-analysis and systematic review of randomized controlled trials. J Cardiothorac Surg 2011;6(1):148. 